\title{
Entre Cisjordanie et Jordanie, l'épreuve du passage frontalier au pont Allenby
}

Between the West Bank and Jordan, the Ordeal of Border-crossing at the Allenby Bridge

Entre Cisjordania y Jordania, las penalidades del paso fronterizo del puente Allenby

\section{Véronique Bontemps}

\section{OpenEdition Journals}

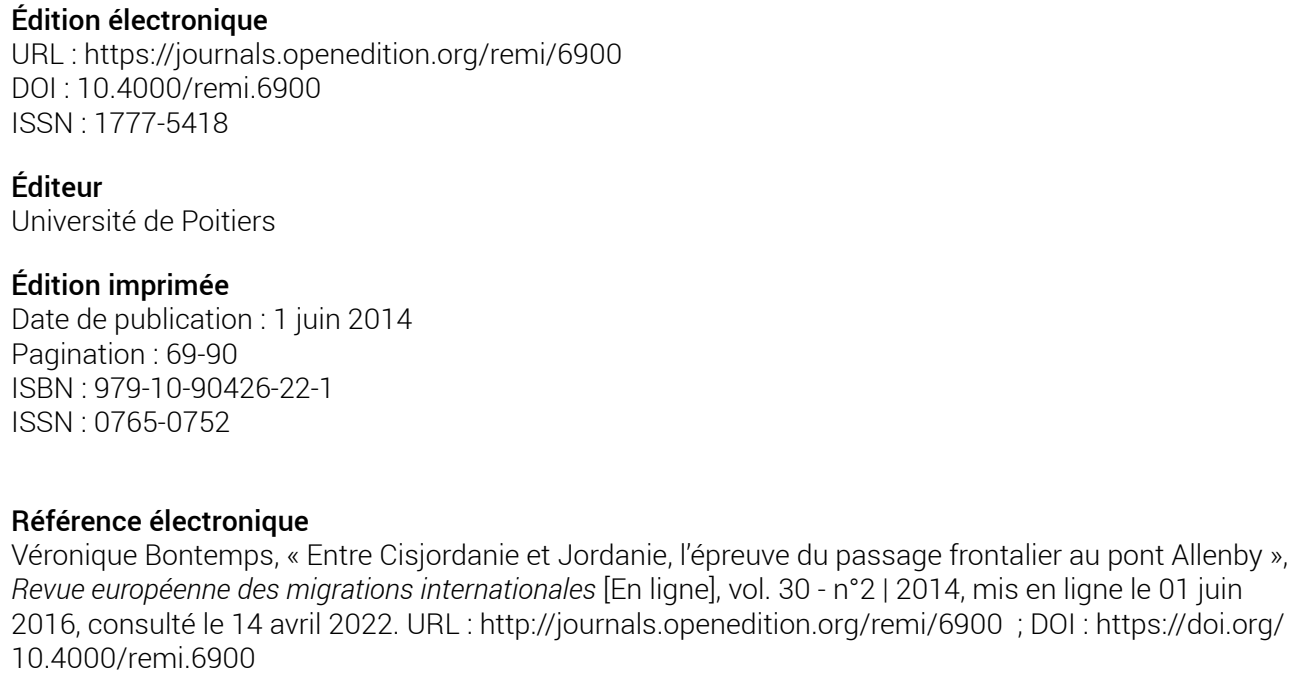

\section{Référence électronique}

Véronique Bontemps, «Entre Cisjordanie et Jordanie, l'épreuve du passage frontalier au pont Allenby », Revue européenne des migrations internationales [En ligne], vol. 30 - $n^{\circ} 2$ | 2014, mis en ligne le 01 juin 2016, consulté le 14 avril 2022. URL : http://journals.openedition.org/remi/6900 ; DOI : https://doi.org/ 10.4000/remi.6900 


\section{Entre Cisjordanie et Jordanie, l'épreuve du passage frontalier au pont Allenby}

\section{Véronique Bontemps ${ }^{1}$}

De nombreux travaux se sont intéressés aux difficultés que les Palestiniens rencontrent régulièrement pour se déplacer, et à la manière dont ces expériences informent bien des aspects de leur vie quotidienne (Parizot, 2009 ; Peteet, 2008 ; Puig, 2013 ; Bontemps, 2012). La traversée du pont Allenby² (point de passage frontalier ${ }^{3}$ entre la Jordanie et la Cisjordanie occupée par Israël) est souvent considérée comme emblématique de ces difficultés qui accompagnent leurs trajets les plus ordinaires. Appelé jisr al-malik Husayn (pont du roi Hussein) par les Jordaniens et ma’abar (passage) al-Karama (" dignité ") par l'Autorité palestinienne, le pont Allenby représente à ce jour le seul point de passage possible pour les Palestiniens vers la Jordanie voisine, ainsi que leur unique " porte de sortie " vers le monde extérieur ${ }^{4}$. Dans cette zone hautement sécurisée, les passagers subissent les contrôles de trois autorités (palestinienne, israélienne et jordanienne) selon un circuit qui s'étire sur plusieurs kilomètres et comprend bus, portiques avec scanners, fouilles et contrôles des papiers. Sur les blogs ou dans les conversations, le " trajet du pont " (rihlat-al-jisr) est unanimement décrit comme une expérience particulièrement pénible ${ }^{5}$.

Le 10 mars 2014 Raed Zuaiter, un juge jordanien d'origine palestinienne est tué lors d'un contrôle au pont Allenby ; il se rendait à Naplouse pour rendre visite à sa famille. Selon les autorités israéliennes, le juge aurait agressé un soldat en tentant de lui dérober son arme ; selon les témoignages des passagers, en revanche, le soldat aurait tiré sur le juge déjà à terre, à la suite d'une vive

\footnotetext{
1 Chargée de recherche CNRS, IIAC-LAU (CNRS - EHESS), 27 rue Paul Bert, 94204 Ivrysur-Seine cedex ; veronique.bontemps@cnrs.fr

2 Les enquêtes de terrain exposées ici ont été réalisées, pour la plus grande partie, dans le cadre d'un post-doctorat à I'IRD (URMIS) entre 2010 et 2012. Je remercie Nicolas Puig, Delphine Moreau et Philippe Bourmaud pour leurs relectures et leurs conseils.

3 Le pont Allenby n'est pas considéré par l'État jordanien comme une frontière internationale.

4 Depuis la deuxième Intifada (septembre 2000), les Palestiniens ne peuvent plus entrer sur le territoire israélien, donc a fortiori se rendre à l'aéroport de Tel Aviv.

5 Voir par exemple le blog du journaliste Daoud Kuttab (www.daoudkuttab.com), et les articles sous le chapeau "Travel Blues".
} 
altercation. L'événement suscita une importante émotion diplomatique ${ }^{6}$ et de nombreux commentaires dans la presse régionale. Dans un article paru quelques jours après, Kuttab écrit : « Toute personne qui traverse le pont [...] sait très bien comment les Israéliens ont, depuis des décennies, bâti une honteuse relation occupant-occupé à la force de leurs fusils ". Arguant que "les opprimés absorbent toutes sortes d'humiliations, simplement pour pouvoir survivre ", il conclut : " le juge jordanien [...] est apparemment entré dans ce bazar (mess) sans le bouclier forgé par toutes ces années d'humiliation, et il n'a pas pu l'accepter " (Kuttab, 2014 [ma traduction]). Ce serait en somme faute d'" habitude" du passage, plus largement faute d'expérience de l'occupation israélienne que le juge Zuaiter serait entré dans cette altercation fatale. Face à la justification israélienne en termes de légitime défense, Kuttab rappelle que le passage du pont comprend une dimension de violence difficilement acceptable pour qui n'en serait pas coutumier.

Dans cet article, j'expose la manière dont les Palestiniens vivent et rendent compte de leur expérience frontalière, lorsqu'ils franchissent le pont Allenby entre la Cisjordanie et la Jordanie. Pour ce faire, je décris en quoi ce passage représente pour eux ce que j'appelle une "épreuve routinière ". J'entendrai ici le mot "épreuve " en deux sens. Tout d'abord, le passage du pont comporte une série de procédures d'accès pour lesquelles il faut se montrer qualifié : ce premier sens correspond à ce que Lussault appelle l'épreuve de franchissement. Dans le contexte contemporain, ces épreuves se sont selon lui généralisées et se déploient de manière emblématique au sein de lieux ultra-sécurisés comme les aéroports, " où I'on doit se soumettre à plusieurs reprises à des contrôles très poussés pour satisfaire une mobilité pourtant élémentaire et banale " (Lussault, 2013 : 181). Ces contrôles sont généralement acceptés comme des contraintes légitimes, nécessaires à l'efficacité et à la sûreté des déplacements. Or la traversée du pont Allenby comporte un enchaînement de procédures que les passagers palestiniens considèrent comme injustifiées et illégitimes : ce qui en fait une épreuve au second sens du terme, c'est-à-dire une expérience pénible, "éprouvante " (au sens de l'anglais ordeal), et qu'ils qualifient de violente".

Mais le passage du pont relève aussi, selon les termes employés par les passagers, d'une forme de "routine ". Si elle n'est pas quotidienne, la traversée vers la Jordanie n'a rien d'exceptionnel, et nombre de Palestiniens ${ }^{8}$ de tous âges et de toutes conditions l'effectuent régulièrement pour de multiples raisons : accomplir des démarches administratives, se soigner dans des hôpitaux souvent mieux équipés qu'en Cisjordanie, voir leur famille ${ }^{9}$, passer quelques

6 Pressé par la rue qui réclamait la démission de l'ambassadeur israélien, le gouvernement jordanien demanda l'ouverture d'une commission d'enquête. Le Premier ministre israélien Benyamin Netanyahou, puis le président Shimon Pérès exprimèrent leurs "regrets".

7 Ce qu'expriment bien les termes arabes appartenant au champ lexical de la souffrance (mu'anâ, ma'sâ, ghalabeh, etc.) que les passagers utilisent lorsqu'ils racontent leur traversée.

8 Selon la police palestinienne, 1219981 personnes ont franchi le pont Allenby en 2011 (583 334 vers la Cisjordanie, et 636647 vers la Jordanie), pour une population d'environ 3 millions d'habitants en Cisjordanie.

9 De nombreux réseaux familiaux palestiniens sont dispersés sur les deux rives du Jourdain. 
jours de vacances ${ }^{10}$ ou encore prendre l'avion depuis la capitale jordanienne. "Routine ", ensuite, parce que le passage se caractérise aujourd'hui par une série de procédures bureaucratico-sécuritaires plus ou moins réglées et codifiées (c'est le sens que le mot rûtîn peut prendre en arabe). "Routine ", enfin, car les voyageurs développent, au fil des passages, des pratiques d'ajustement pour tenter de maîtriser davantage le déroulement de la traversée et la rendre " acceptable ". Par l'expression volontairement paradoxale d'" épreuve routinière " (qui s'inspire de l'acception vernaculaire des termes $d^{\prime}$ " épreuve " et de " routine $\left.{ }^{11}\right)$, je cherche ainsi à rendre compte de l'imbrication entre une certaine forme de violence et l'aspect pourtant habituel qui caractérise aujourd'hui, pour beaucoup de Palestiniens, le passage du pont Allenby ${ }^{12}$.

Ce sont les différents aspects de cette "épreuve " que je souhaite décrire dans cet article. Je m'appuie sur des observations effectuées lors de mes passages du pont avec des Palestinien(ne)s, en moyenne une fois par mois entre 2010 et 2012 (puis de manière moins fréquente jusqu'en 2014). J'ai complété ces observations par des récits d'expériences ${ }^{13}$ recueillis dans plusieurs lieux : au pont, à Jéricho, Naplouse, Ramallah et Amman. Les descriptions qui suivent sont donc faites essentiellement du point de vue des " traversants ", et non de celui du pouvoir et de ses personnages, prestataires ou représentants des autorités de contrôle. Si ce parti pris constitue une limite, il me semble pourtant qu'une description au plus proche de l'expérience des passagers permet d'apporter un éclairage sur l'épaisseur que prennent très concrètement les zones frontalières pour certaines populations (" une zone spatio-temporelle extraordinairement visqueuse ", écrit Balibar) : un lieu où I'on passe (et où l'on perd) du temps, où I'on peut à tout moment se trouver coincé ; un lieu "où l'on revient sans cesse se heurter " (Balibar, 1997 : 379).

À partir d'un rappel historique, je présenterai d'abord comment s'est installée, avec le temps, une " situation de frontière " (Agier, 2013) au pont Allenby. Je proposerai ensuite une description des différentes étapes pour montrer que le passage du pont possède une dimension de violence, qui opère simultanément à plusieurs niveaux. J'évoquerai enfin ce que j'appelle des " routines " de franchissement, qui relèvent à la fois de tactiques individuelles et de pratiques d'entraide entre passagers.

\footnotetext{
10 Peu de pays accordent un visa aux Palestiniens.

11 Je suis ici les registres que développent les Palestiniens eux-mêmes quand ils parlent de l'épreuve comme expérience pénible, et de la routine comme habitude (et/ou tracas bureaucratico-sécuritaire). L'expression a valeur plus descriptive qu'analytique, et ne fait donc pas référence à la sociologie pragmatique ou " des épreuves " (selon la formulation de, entre autres, Lemieux, 2007).

12 Tout comme d'autres situations de passage frontalier auxquelles ils sont régulièrement confrontés (notamment le passage des checkpoints). Je m'inspire ici d'un article de Perdigon (2010: 981) où il s'interroge sur " la désorientation qu'on peut subir, en tant qu'ethnographe, dans la confrontation avec des formes de violence qui n'indiquent pas tant une rupture du quotidien que sa continuité ".

13 Sauf indication contraire, les entretiens ont été effectués en arabe et traduits par moi.
} 


\section{La mise en place d'un espace frontalier au pont Allenby}

\section{Passer le pont « au début " (bil-awwah)}

Le pont Allenby a été construit en 1918 par le général britannique Edmund Allenby, sur un ancien pont datant de la période ottomane. II n'a pas toujours représenté un espace frontalier: sous I'Empire ottoman, les deux rives appartenaient à une même entité territoriale socialement intégrée. Après la Nakba ${ }^{14}$ de 1948, la conférence de Jéricho décida de l'union administrative des deux rives pour former le Royaume hachémite de Jordanie ${ }^{15}$. C'est donc I'occupation israélienne de 1967 qui interrompit la liberté de circulation entre les deux rives du Jourdain. Après une fermeture totale pendant plusieurs mois, les Palestiniens furent autorisés à se rendre en Jordanie via deux "ponts " tenus, de part et d'autre du Jourdain, par les armées israélienne et jordanienne : le pont Damia pour le nord de la Cisjordanie, et le pont Allenby pour le sud.

Photo 1 : Le pont Allenby en 1968, photographie encadrée dans un bureau de " l'administration des passages " côté palestinien

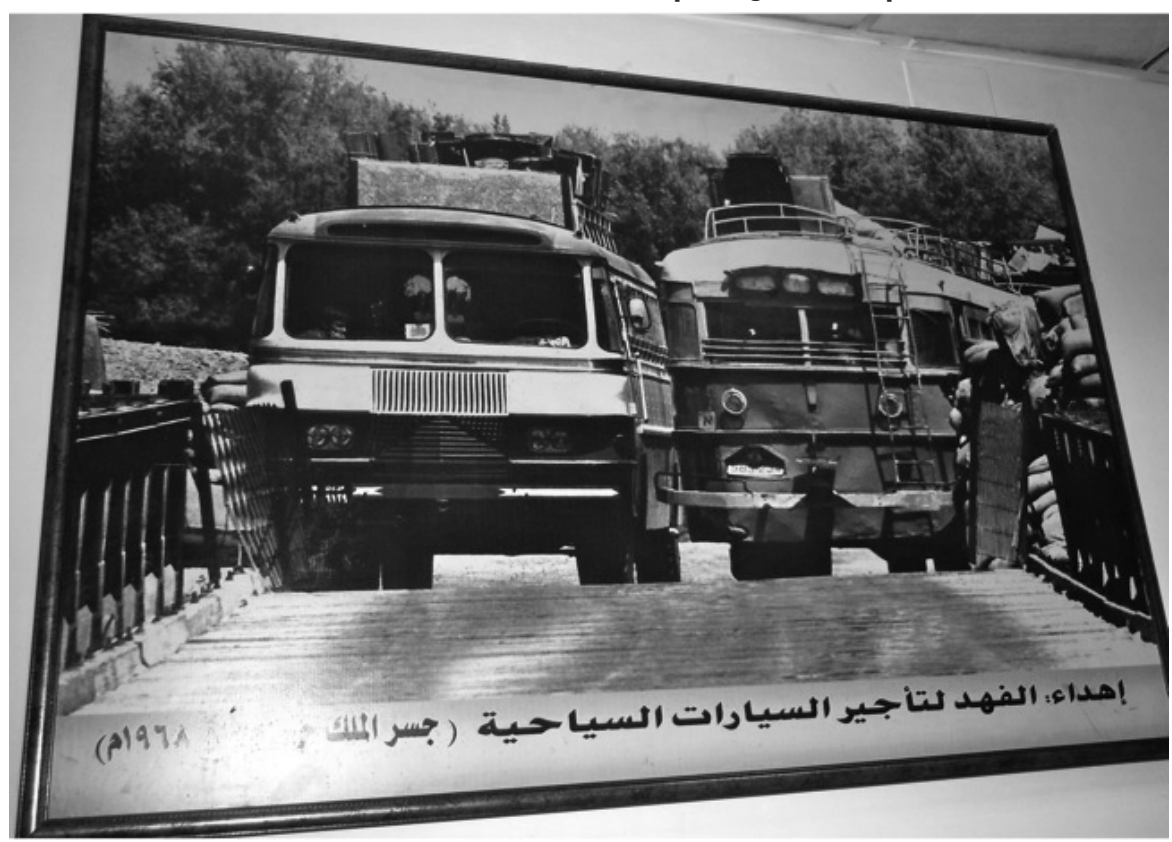

Crédit : Véronique Bontemps.

14 Nom donné par les Palestiniens à la partition de la Palestine mandataire et à la création d'Israël, ainsi qu'à la fuite de plusieurs centaines de milliers de Palestiniens en 1948-1949.

15 La Cisjordanie resta considérée par la Jordanie comme une partie de son territoire jusqu'à la rupture des liens administratifs et juridiques décidée par le roi Hussein le 31 juillet 1988, et ce même après I'occupation israélienne de 1967. 
Le passage des ponts à ses "débuts " (bil-awwal) fut d'emblée associé à l'expérience d'une grande violence. Les témoignages décrivent une multitude de procédures peu claires, une impression générale de désordre (fawda) allant de pair avec le caractère peu aménagé de l'espace frontalier : un pont en bois branlant, des auvents et baraquements en lieu et place de poste-frontière. Sont évoquées des procédures de filtrage fortement prohibitives, intrusives et humiliantes (fouilles corporelles systématiques). Ces souvenirs de voyageurs palestiniens apparaissent d'autant plus frappants que ce sont, précisément, des souvenirs de "premières fois ": ils faisaient l'expérience, tout à la fois, de l'occupation militaire israélienne et d'un nouveau régime frontalier.

Dans les années 1980, la mairie de Jéricho décida conjointement avec l'administration civile israélienne d'installer une istiraha (zone d'attente) à Jéricho, à quelques kilomètres du pont Allenby : il s'agit d'un espace de regroupement pour les passagers en provenance des différentes villes de Cisjordanie. Avec la fermeture du pont Damia en 1991, cette istiraha qui s'agrandit avec les années devint la première étape obligatoire du voyage vers la Jordanie.

En 1994, il fut décidé lors des accords d'Oslo que les terminaux de départ et d'arrivée seraient tenus conjointement par les Israéliens et l'Autorité palestinienne - Israël restant en charge de la " sécurité " du passage, "indirectement, et de manière invisible ${ }^{16}$. Cependant, après la deuxième Intifada puis dans la mouvance du nouvel ordre sécuritaire post-11 septembre 2001, la police palestinienne dut se retirer du poste-frontière pour s'installer à Jéricho, à " I'administration des passages " située à quelques kilomètres du pont. Elle opère aujourd'hui depuis l'istiraha qui représente de facto, depuis les rénovations entreprises par l'Autorité palestinienne ${ }^{17}$, le poste-frontière palestinien.

De nos jours, les personnes évoquant la traversée s'accordent à dire que celle-ci est " mieux organisée ". Depuis 2009, le pont est ouvert de 7 heures du matin à minuit, sauf les week-ends ${ }^{18}$. Le voyage en Jordanie $n^{\prime}$ a plus rien d'exceptionnel et si les premiers passages continuent de marquer les mémoires, les espaces frontaliers se sont pérennisés et les procédures de contrôle affinées. Comparant le passage des ponts " au début " avec la situation actuelle, Samia, vieille dame de la bourgeoisie naplousaine, me dit :

"C'était un voyage très difficile. Une fois qu'on l'avait fait, on ne voulait plus voyager. Aujourd'hui, on n'a toujours pas envie de voyager, note bien! [Elle rit]. Mais avec toutes les difficultés qu'il y a, c'est toujours un peu mieux qu'avant. Au moins, il y a un semblant d'ordre " (entretien avec Samia, Naplouse, 2012).

\footnotetext{
16 Le passager entrant dans le terminal ne devait voir qu'un policier et un drapeau palestiniens. Concrètement, pourtant, un agent israélien se tenait derrière une glace sans tain pour contrôler les documents que le policier palestinien lui remettait par un tiroir; les miroirs étaient placés " de telle sorte que la sécurité israélienne [...] pouvait surveiller, sans être vue, non seulement les passagers palestiniens, mais aussi le personnel de police palestinien »(Weizman, 2007 : 140 [ma traduction]).

17 L'Autorité palestinienne reprit en 2010 la gestion de l'istiraha à la mairie contre compensations financières. La salle de départ a été agrandie, mieux climatisée ; les formalités de police y ont été installées et informatisées ; de nouveaux bureaux y ont été mis en place, ainsi qu'une salle d'arrivée.

18 C'est-à-dire les vendredis et samedis, où le pont n'est ouvert que la mi-journée.
} 
L'augmentation du nombre des passagers (en hausse constante depuis 2010) et les différents aménagements réalisés ont modifié les perceptions qui restaient associées aux premiers passages. Ces évolutions n'ont cependant pas supprimé la violence, encore moins l'inconfort de la traversée : ils ont plutôt participé d'une normalisation et d'une banalisation de cette violence, qui marque aujourd'hui encore l'expérience des voyageurs palestiniens.

\section{Passer le pont aujourd'hui : les étapes du trajet aller et retour}

Le trajet d'un passager palestinien allant de Naplouse à Amman (environ 150 kilomètres) se décompose en plusieurs étapes. Tout d'abord, il lui faut se rendre à I'istiraha de Jéricho : c'est là que la police palestinienne organise le départ vers le terminal israélien ${ }^{19}$. Des numéros sont attribués à chaque voyageur à son entrée dans I'enceinte de I'istiraha. Devant la salle de départ, un porteur dépose les valises dans un camion qui les acheminera séparément. Puis les passagers achètent leurs billets de bus, paient leur taxe de sortie $e^{20}$ et $\mathrm{s}^{\prime}$ asseyent dans la salle pour attendre l'appel de leur numéro ${ }^{21}$.

\section{Photo 2 : La salle de départ de l'istiraha}

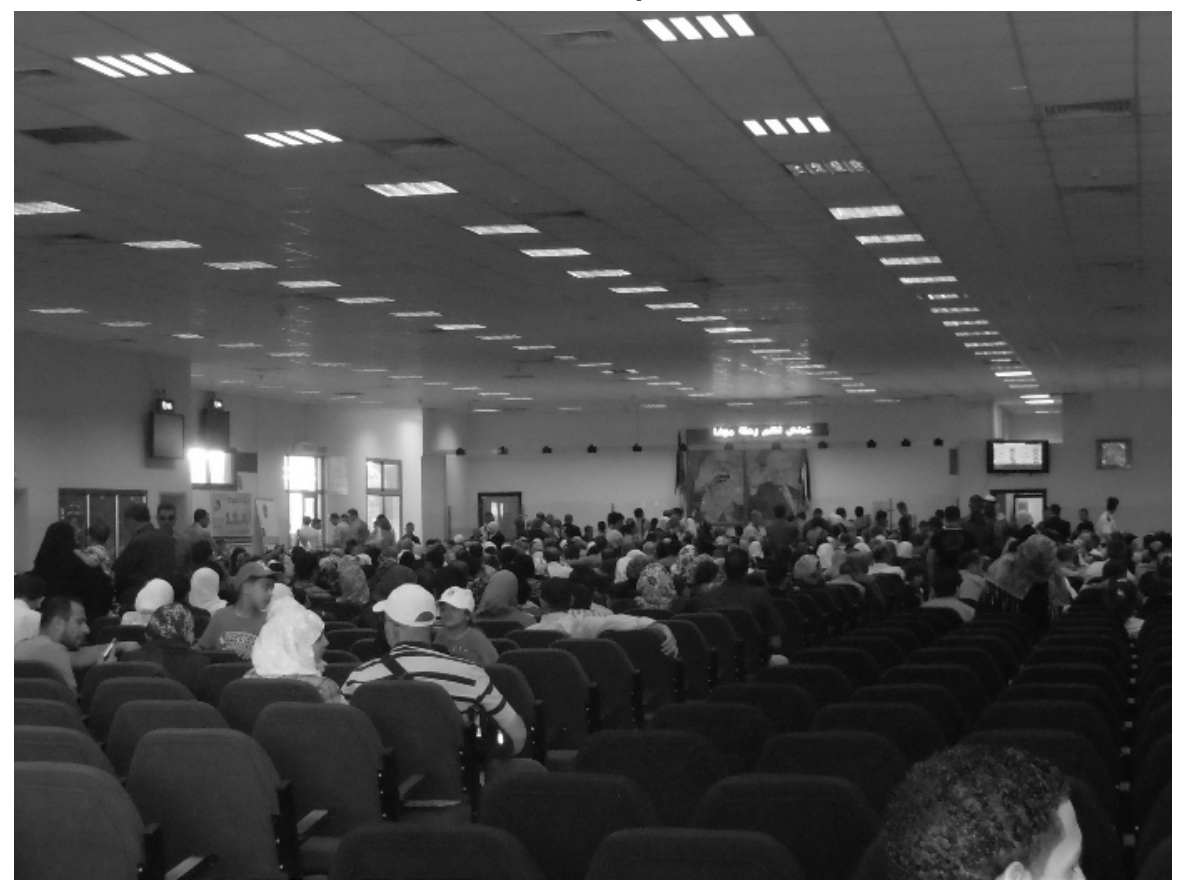

Crédit : Véronique Bontemps.

19 Soixante-trois policiers travaillent en permanence à l'istiraha, où sont également installés les autres appareils de sécurité de l'Autorité palestinienne : les renseignements (mukhabarât) et les renseignements militaires (istikhbarât).

20 Le montant de cette taxe est versé à l'avance par l'Autorité palestinienne aux Israéliens.

21 La salle de départ de l'istiraha a une contenance de 1000 sièges. 
Une fois les guichets passés et le tampon palestinien apposé sur les cartes $\mathrm{d}^{\prime}$ embarquement ${ }^{22}$, les passagers montent dans un premier bus qui s'arrête un kilomètre plus loin à un checkpoint appelé al-'alami ${ }^{23}$. Ils passent un portique de sécurité sous le regard d'un soldat israélien, et embarquent dans un deuxième bus qui attend à vide ; ce bus franchit le checkpoint dit " porte 142 " ${ }^{24}$ où il entre dans la zone frontalière ${ }^{25}$. Après un deuxième checkpoint, il gagne le " pont israélien ${ }^{26}$.

Au signal d'un employé (souvent un soldat), les passagers descendent et se placent dans la queue devant la salle de départ. Ils passent par deux portillons de sécurité, puis attendent aux guichets pour les formalités. Ils prennent ensuite un troisième bus qui les emmène à la " place des bagages ${ }^{27}$; ils y récupèrent leurs sacs et montent dans un quatrième bus qui traverse le pont. Une fois du côté jordanien ${ }^{28}$, ils descendent (une fois qu'on les y a autorisés) devant la " salle d'arrivée des Arabes ${ }^{29}$. Ils prennent un ticket à l'entrée et attendent leur tour. Ils effectuent les formalités au guichet, puis paient une taxe d'entrée de 10 dinars (environ 10 euros) avant de récupérer leurs bagages. À la sortie, ils peuvent choisir entre taxi privé, taxi collectif ou bus pour rejoindre leur destination jordanienne (souvent Amman ou I'aéroport).

À leur retour, les passagers gagnent le " secteur des Arabes " au " pont jordanien ". Ils déposent leurs bagages sur un tapis roulant, puis accèdent à un guichet; ils y achètent leur billet de bus et entrent dans la salle de départ. Ils y attendent que leur numéro soit appelé puis, " carte de départ " en main, ils récupèrent leur bagage et montent dans le bus qui les emmène au " pont israélien ". Sur les quelques kilomètres qui séparent les deux terminaux, le bus subit plusieurs contrôles. Avant d'entrer en territoire israélien, il s'arrête devant un barrage. Plusieurs bus stationnent parfois à la file sur la structure du pont, dans l'attente qu'un soldat israélien actionne la barrière. Puis, nouvel arrêt devant un abri où les passagers doivent descendre. Les cartes d'identité sont contrôlées, le bus fouillé, puis les voyageurs remontent pour être emmenés au

22 L'Autorité palestinienne ne tamponne pas le passeport palestinien, pas plus que l'État jordanien au pont Allenby.

23 Ce checkpoint a été instauré en 1994, après les accords d'Oslo : il signale la sortie de la zone A (prévue par les accords comme entièrement sous autorité palestinienne) pour entrer dans la zone C (sous contrôle israélien).

24 Les Palestiniens de Jérusalem ne passent pas par l'istiraha et entrent au pont directement par la porte 142.

25 C'est une zone militaire fermée identifiable à un no man's land, où se situent les terminaux israélien et jordanien. Au milieu coule le fleuve Jourdain (aujourd'hui un maigre cours d'eau, quand il n'est pas asséché) enjambé par une structure en béton, le " pont " à proprement parler.

26 Depuis 1994, les terminaux israéliens sont gérés par I'Israeli Airport Authority, une entreprise nationale qui travaille en coopération avec le ministère de l'Intérieur et I'armée. Depuis quelques années, elle engage également des compagnies privées pour I'accueil des passagers.

27 II s'agit d'un espace de stockage où les valises sont placées sur des étagères.

28 Le côté jordanien du pont est opéré depuis les années 1970 par un département de la police jordanienne, la "Sûreté générale " (al-amn al-'âm), appelé l' " administration de la sécurité des ponts " (idârat amn al-jusûr).

29 Le terminal jordanien est divisé en deux secteurs : le " secteur des Arabes " (qism $\left.a l-{ }^{\prime} a b\right)$ pour les passagers munis d'une carte d'identité palestinienne, et le " secteur tourisme " (qism al-siyâhha). 
" pont israélien ", derrière un dernier checkpoint. Les passagers attendent le signal d'un agent armé pour descendre ; ils s'engagent avec leurs bagages dans une allée en chicane menant à des tapis roulants, où ils déposent leurs valises. Celles-ci sont étiquetées d'un code-barres également apposé sur le passeport. À un premier guichet, les passagers présentent leurs passeports sur lesquels un employé met un numéro indiquant si une fouille (taftîsh) doit être effectuée.

\section{Photo 3 : Le checkpoint d'al-'alamî}

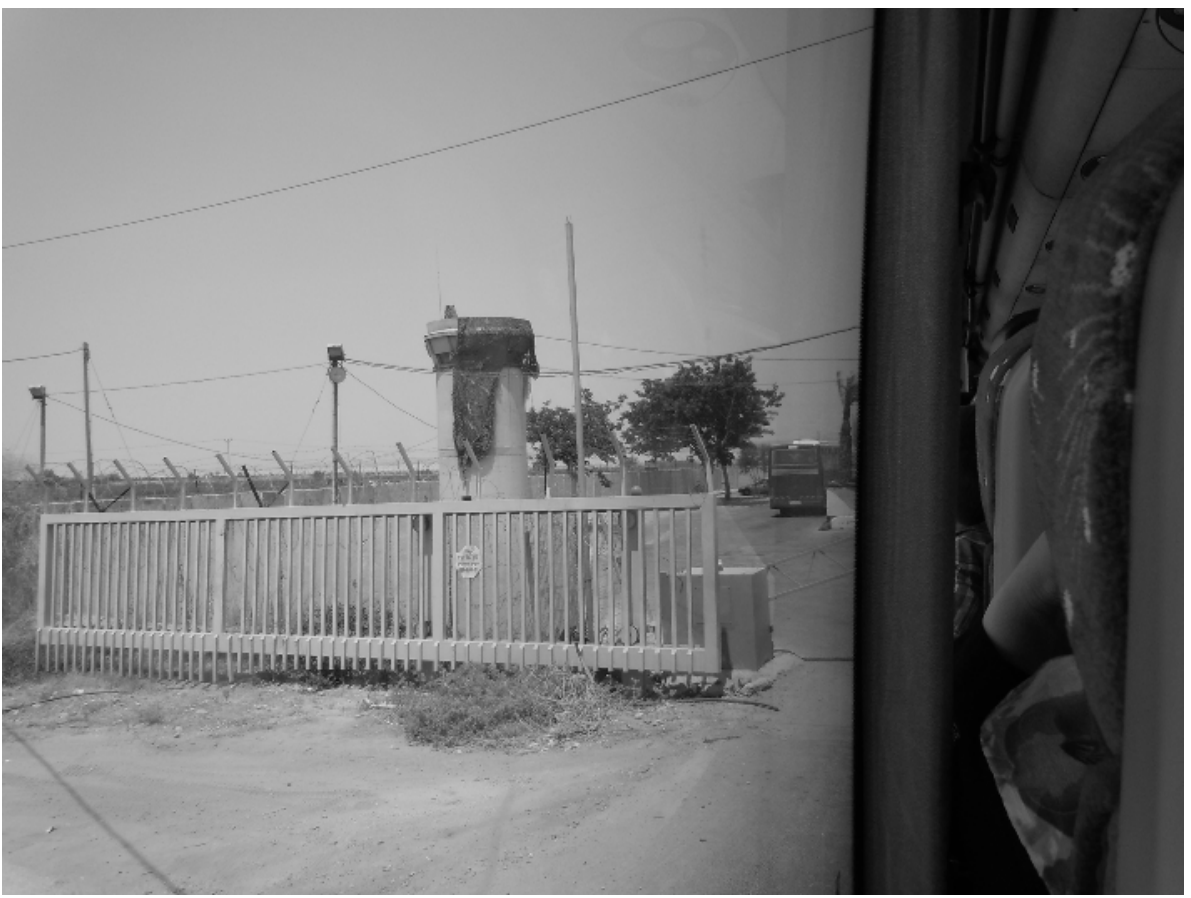

Crédit : Véronique Bontemps.

À l'intérieur de la salle, un nouveau dispositif en chicane mène à deux portiques et scanners. Les passagers posent leurs bagages à main, passent le portique ; certains sont emmenés pour fouille et interrogatoire. Puis ils se répartissent dans les queues aux guichets $d^{\prime} e^{\prime} t^{2} e^{30}$. Ils présentent leurs papiers : après contrôle et parfois quelques questions, ils espèrent que leur passeport sera tamponné et qu'ils pourront avancer vers la sortie. Certains sont priés de s'asseoir sur des sièges en métal disposés en carré devant les guichets, et d'attendre qu'on les appelle pour des vérifications plus poussées. Enfin, si le code-barres l'indique, les valises sont éventuellement fouillées. Les passagers passent enfin par la douane israélienne puis prennent le bus pour Jéricho.

30 Des panneaux signalent en hébreu, anglais et arabe que les porteurs de " passeports étrangers " doivent emprunter la file de gauche, les " cartes d'identité palestiniennes " celle du milieu, et les " cartes d'identité de Jérusalem " celle de droite. 


\section{Photo 4 : Les bus qui attendent sur le pont}

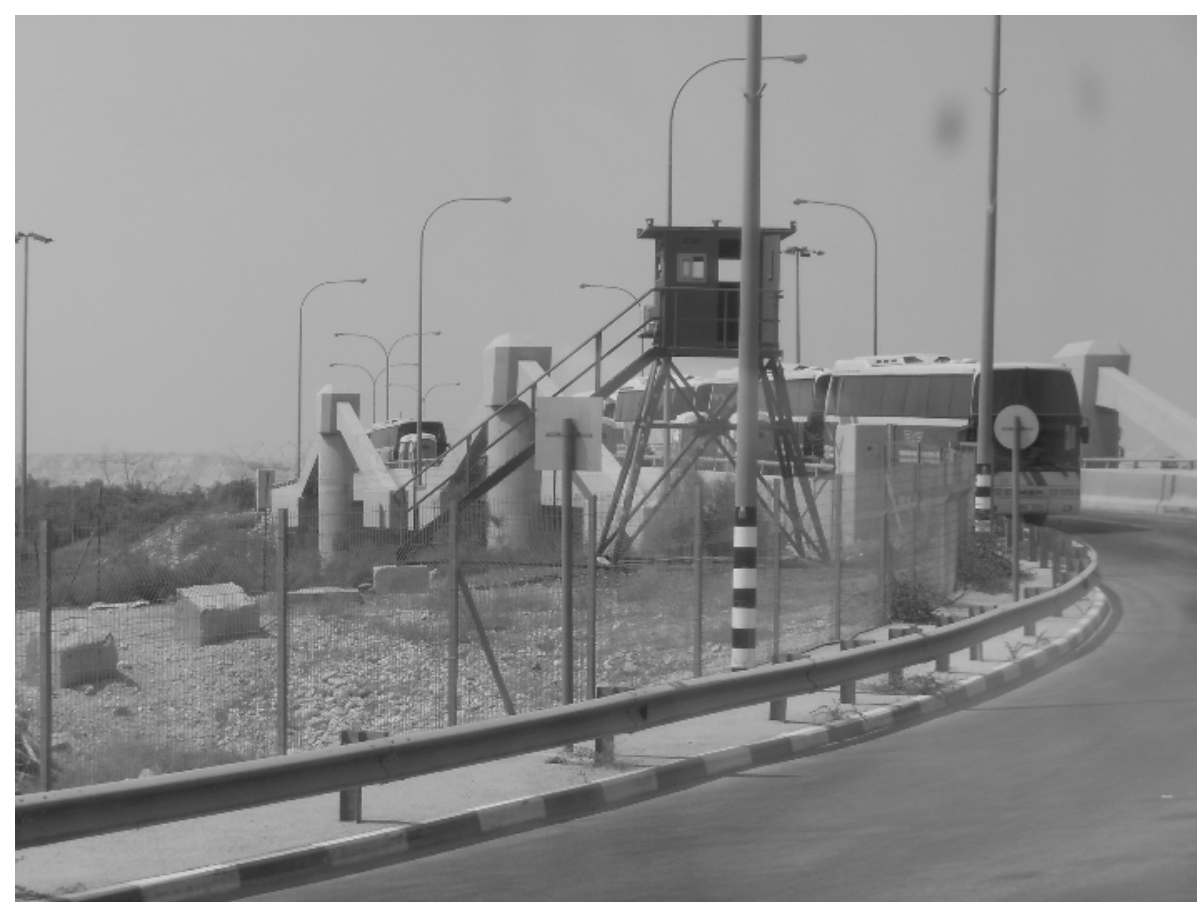

Crédit : Véronique Bontemps.

Photo 5 : L'entrée du terminal israélien d'arrivée

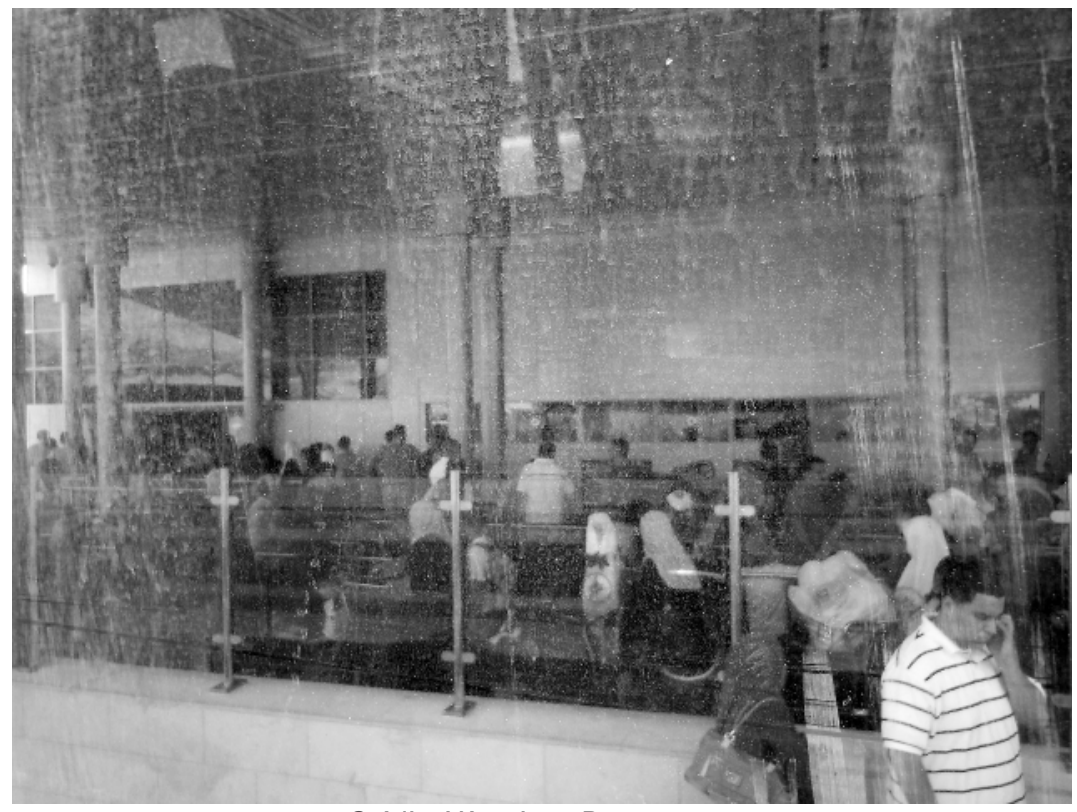

Crédit : Véronique Bontemps. 
Dans la salle d'arrivée palestinienne, les passagers sont reçus par des policiers palestiniens. Ils enregistrent leur entrée en Cisjordanie, récupèrent leurs bagages préalablement scannés, subissent éventuellement une fouille des douanes palestiniennes, puis sortent vers la gare routière d'où les taxis collectifs les conduiront vers leurs différentes destinations.

\section{L'attente}

La violence du passage réside, à un premier niveau, dans la répétition des contrôles, et les temps d'attente souvent très longs et pénibles (parfois dix ou douze heures en été). Cette perte de temps n'est pas seulement économiquement coûteuse, elle a aussi une portée symbolique : le temps consacré à la traversée du pont est un moment de confrontation au pouvoir de l'occupant, les heures passées à attendre renvoyant les passagers palestiniens à leur absence de souveraineté (Parizot, 2009 ; Bontemps, 2012). À cet aspect symbolique s'ajoute la violence matérielle des conditions dans lesquelles se déroule l'attente :

"Tu prends un bus, tu peux être arrêté et attendre comme ça. Une fois, j'ai compté quinze bus devant moi ! Quand il y a quinze bus, tu peux attendre trois heures, et en été, dans la vallée du Jourdain... il peut faire cinquante degrés... Si tu as des enfants, si tu es malade, si tu es diabétique et que tu as besoin de soins... ils ferment le bus, tu n'as pas le droit de descendre ! " (entretien avec S.H., enseignant à l'université de Naplouse, 2011).

Samia signale qu'il est sage de se munir d'une vieille valise et d'éviter d'y mettre des objets fragiles :

" Jusqu'à maintenant, ils jettent les sacs d'une manière... Si tu as une jolie valise, tu te dis que c'est franchement pitié (harâm) de la prendre au pont ! Parce qu'on m'a déjà cassé des sacs... [Elle poursuit:] Tu changes de voiture 1 million de fois avant d'arriver à Amman... Et tu paies comme si tu prenais un avion pour aller en Amérique ! " (entretien avec Samia, Naplouse, 2012).

Dans les entretiens, les passagers évoquent les bus bondés d'où il est interdit de descendre, les salles mal climatisées, la manière dont sont manipulés les bagages, l'attitude des autorités de contrôle tant israéliennes que jordaniennes, le coût du trajet (qui s'élève à environ 65 euros par personne) : " toutes ces petites choses qui sont humiliantes ", selon la formule de S.H.

\section{L'incertitude et l'arbitraire}

Le caractère éprouvant du passage réside également, pour mes interlocuteurs, dans la dimension d'incertitude qu'il comporte : incertitude sur la possibilité du voyage ; incertitude sur la durée du trajet, ainsi que sur son issue. Si les passagers qui possèdent une carte d'identité palestinienne sont en principe assurés de pouvoir entrer en Cisjordanie, ils n'échappent pas nécessairement à un interrogatoire dont le déroulement leur reste obscur. Pour les Palestiniens munis de documents étrangers, l'entrée en Cisjordanie n'est pas acquise. Au guichet, ils sont interrogés sur les raisons de leur visite, leur histoire familiale, etc. Puis ils devront attendre l'appel de leur nom, pour une éventuelle enquête plus poussée menée par des employés de la police, du ministère de l'Intérieur, voire des services de renseignements ou de l'immigration israélienne ; cette enquête peut se solder par un refoulement dont les motifs ne sont pas 
précisés. En outre, le passage du pont est soumis à un agenda politique qui est susceptible de bouleverser les horaires, de bloquer les bus ou de provoquer la fermeture du pont. Les autorités israéliennes refusent aussi parfois le passage de voyageurs, si elles estiment par exemple que leurs documents ne sont pas suffisamment lisibles.

\begin{tabular}{l} 
Alors que je suis au guichet [israélien], j'entends un homme \\
parlementer en anglais avec une employée : on lui refuse le passage, \\
car sa carte d'identité a pris l'eau. Elle lui explique qu'il doit apporter \\
un permis spécial. C'est vendredi, et le pont va bientôt fermer. Le \\
jeune homme demande à retourner faire le permis à Jéricho, et qu'on \\
le laisse ensuite revenir en taxi : il a son avion pour Dubaï le jour \\
même. Mais c'est peine perdue, il doit faire demi-tour ${ }^{31}$. \\
\hline
\end{tabular}

Il faut enfin compter avec les interdictions de sortir du territoire israélien ou d'entrer en Jordanie, que les personnes concernées ne découvrent parfois que sur place. Les passagers se trouvent sans aucune prise sur ces retards ou ces refoulements : un pouvoir discrétionnaire s'exerce sans qu'il y ait possibilité d'un quelconque recours.

Le géographe Michel Lussault s'est intéressé à ce qu'il voit comme une généralisation contemporaine des techniques de contrôle d'accès " qui s'imposent aux individus qui veulent franchir " (Lussault, 2012 : 71). II ramène ces procédures à trois grandes orientations : queuing (ou gestion de l'attente), filtrage et traçage. La traversée du pont représente bien aujourd'hui un circuit apparemment balisé où l'on retrouve ces protocoles codifiés : espaces réservés dans les salles, dispositifs en chicane, bas-côtés des routes dévolus à la queue des bus (queuing) ; portiques équipés de scanners, tapis roulants pour les bagages (filtrage d'accès) ; suivi des bagages au moyen de code-barres, surveillance des individus par des caméras (traçage). Depuis quelques années, les travaux en cours au pont israélien visent à transformer progressivement son aspect en " terminal " semblable à ceux des aéroports internationaux. Pourtant, alors que la soumission aux contrôles (parfois répétés) dans ces terminaux provient de I'acceptation tacite des règles du jeu par les passagers, ils ne sont pas perçus au pont comme légitimes : les voyageurs palestiniens considèrent au contraire les conditions du passage comme injustifiées et comme une atteinte à leur dignité (Bontemps, 2012). Farida, militante pour la campagne KARAMA (" dignité ") ${ }^{32}$, m'explique :

"C'est justifié que les frontières soient sécurisées, ça tout le monde le comprend. Mais là, les fouilles tu les subis plus de sept fois, et ça, ce n'est pas possible... sauf si tu penses que l'autre n'est pas un être humain " (entretien avec Farida, Ramallah, juin 2014).

Dans ce contexte, les récentes " améliorations " et l'aspect apparemment réglé des procédures sont vus par les passagers comme une façade cachant le

\section{Observation juillet 2011.}

32 La campagne KARAMA, lancée par des personnalités de la société civile palestinienne à partir de 2008, revendique la liberté de mouvement des Palestiniens, notamment vers la Jordanie. Elle demande qu'ils puissent s'y rendre en un minimum de temps, avec un minimum de formalités, en payant un minimum d'argent. Concrètement, elle exige qu'ils puissent traverser le pont Allenby, dans leur voiture, à n'importe quelle heure du jour et de la nuit. 
caractère oppressif des relations de pouvoir qui président au passage ${ }^{33}$. Du fait de la démultiplication des étapes et des contrôles, le dispositif policé se grippe : des goulots d'étranglement se créent les jours d'affluence ${ }^{34}$. Les bagages s'entassent et se perdent, les files d'attente s'allongent. Par ailleurs, les acteurs du passage ne sont pas toujours bien identifiés par les voyageurs ${ }^{35}$. Ces derniers ont le sentiment que les procédures de contrôle, sous prétexte d'une garantie de " sécurité ", auraient plutôt à voir avec une entreprise voulue de déstabilisation, voire d'humiliation systématique ${ }^{36}$. Ainsi pour Samia :

"Cela n'a pas de sens. Si je veux aller d'un pays à un autre, je comprends qu'on me pose des questions à la frontière, que je montre mon passeport... Pas qu'on me fasse attendre pendant des heures sans raison! Et si je faisais le trajet dans ma voiture, qu'est-ce que ça changerait? Ils n'ont qu'à regarder le passeport, regarder la voiture, et c'est tout, c'est ça la sécurité !" (entretien avec Samia, 2012).

\section{Savoir franchir le pont : compétences et " routines ${ }^{37}$ "}

Malgré l'étroitesse des marges de manœuvre, certains voyageurs mettent en place des tactiques qui visent à organiser au mieux le franchissement de I'espace frontalier. En partant du sens que les passagers palestiniens donnent à ce mot, je propose d'appeler ces pratiques de franchissement des "routines " (rûtîn). J'entendrai donc ici la " routine " comme un ensemble de compétences pratiques, acquises à travers un processus de familiarisation et d'appropriation par l'usage, qui donne une dimension d'aisance dans l'accomplissement d'une tâche. Ainsi conçue, cette notion permet de rendre compte des savoir-faire ou manières d'agir acquis au fil des passages du pont. Plus ou moins élaborés et affichés, ils jouent sur plusieurs registres. Certains sont du domaine de compétences indispensables au franchissement ; d'autres constituent des actions rassurantes ou même ludiques, donnant le sentiment de déjouer l'arbitraire du passage et de le maîtriser quelque peu ; d'autres encore permettent d'habiter les heures d'attente et d'ennui. Certains, enfin, tendent à restaurer une dignité mise à mal lors du passage. De la mise en œuvre de ces savoir-faire découlent des

\footnotetext{
33 On peut considérer que les travaux côté israélien, en cours au moment de l'enquête, s'inscrivent dans la même tendance que la privatisation des checkpoints frontaliers entre Israël et la Cisjordanie et leur réaménagement en terminaux, qui vise à " réduire la friction [...] et améliorer la qualité du service en maintenant la qualité du contrôle " (Unité d'information et de recherche de la Knesset, 2006, cité par Havkin, 2011 : 52). Selon Havkin (2011 : 53), cette politique représente une tentative de polissage, qui masque le caractère oppressif des relations de pouvoir intervenant aux checkpoints et les nouvelles formes de domination et d'arbitraire auxquelles elles donnent lieu.
}

34 Les dimanches et mercredis, jours de retour de 'umra. Moins long que le Hajj, ce " petit pèlerinage " peut être effectué presque tout au long de l'année. Il y a aussi beaucoup de monde les week-ends et pendant les vacances d'été.

35 Notamment les employés de compagnies privées qui tendent à remplacer les soldats pour les contrôles aux guichets.

36 Les autorités jordaniennes n'échappent pas à cette critique : leur attitude est même souvent perçue comme plus violente encore que celle des Israéliens dans la mesure où, comme le dit Nader : "le coup est pire quand il vient d'une main arabe " (entretien avec Nader M., Naplouse, 2014).

37 La notion de "routine ", essentiellement mobilisée en sociologie du travail (Voir Breviglieri, 2004 ; 2006.) comprend généralement deux versants : une dimension de répétition, voire d'automatisme ; et une dimension tactique et créative, " des capacités ordinaires à bricoler et "faire avec" " (Certeau, 1990 ; Breviglieri, $2004: 21)$. 
formes de réappropriation de l'espace-temps de la traversée, avec laquelle on se familiarise, malgré l'incertitude du passage et l'attitude des autorités de contrôle.

\section{Organiser les départs}

Dans un premier temps, il s'agit de prévoir. Pour franchir le pont, les voyageurs palestiniens doivent se munir : de leur passeport valide (jordanien ou palestinien $^{38}$ ) ; de leur carte d'identité (hawiyya) de Cisjordanie ou de Jérusalem ${ }^{39}$; d'un permis (tasrîh) de sortie s'ils utilisent leur passeport jordanien ${ }^{40}$; de leur " carte du pont " délivrée par les autorités jordaniennes ${ }^{41}$. Cette liste est néanmoins susceptible d'être modifiée, comme me le raconta par exemple Samia :

"Un jour, je suis partie pour Amman. [...] Je faisais la queue, ils [les Israéliens] m'ont dit: "Attends un peu, on va t'apporter ton passeport". II y avait peut-être soixante-dix personnes avec moi. On a attendu une heure, deux heures, trois heures... À la fin de la journée [...] ils nous ont dit: "Vous devez faire demi-tour". [...] Et pour quelle raison? Apparemment, la veille au soir, une loi avait été promulguée comme quoi on ne pouvait passer qu'avec son passeport palestinien. [...] Et celui qui voyage le lendemain, comment il peut savoir? " (entretien avec Samia, Naplouse, juillet 2012).

La préparation des bagages demande aussi de savoir ce que l'on peut transporter ou pas avec soi. Certaines denrées (comme l'huile d'olive par exemple) ne sont autorisées qu'en quantité limitée ; les appareils électriques ou les ordinateurs portables peuvent être soumis à taxation ; le lait infantile ou le café posent problème lors du passage aux rayons X. Enfin, il faut vérifier qu'il n'y aura pas de modification des heures d'ouverture ou de fermeture. Les risques de changement d'horaires ou de blocages des bus créent un principe d'incertitude que Romani appelle « hétérochronie $n^{42}$. Cela oblige les voyageurs à encore plus de prévoyance : la plupart d'entre eux partent un jour à l'avance s'ils ont un avion à prendre, et souvent très tôt le matin ; ils espèrent ainsi attraper le premier bus et gagner Amman en fin de matinée. D'autres au contraire préfèrent la fin d'après-midi, afin d'éviter l'affluence des heures matinales. Les voyageurs sont du reste encouragés par les autorités palestiniennes à étaler leurs horaires de départ sur l'ensemble de la journée pour " relâcher la pression " sur les policiers qui gèrent l'attente à l'istiraha ${ }^{43}$.

38 Après 1988, les Palestiniens de Cisjordanie perdirent leur nationalité jordanienne, mais gardèrent le droit à un passeport jordanien comme " document de voyage ". Beaucoup l'ont conservé, même après l'émission par l'Autorité palestinienne de ses propres passeports après 1994.

39 Ces cartes d'identité sont aujourd'hui émises par l'Autorité palestinienne, mais toujours contrôlées par Israël.

40 Les Palestiniens voyageant avec un passeport jordanien doivent impérativement se faire établir un permis de sortie. C'est aujourd'hui une simple formalité, dont le montant s'élève à 50 shekels (10 euros).

41 La " carte de statistique des ponts ", verte ou jaune selon que le porteur possède ou non la nationalité jordanienne, est un document imposé par les autorités jordaniennes en 1983 pour le passage du pont Allenby (Ababsa et Daher, 2011 ; Bonnefoy, Bontemps et Guignard, 2012).

42 II entend par là : " la soumission des rythmes sociaux et individuels à la décision israélienne et non pas à celle des acteurs palestiniens " (Romani, 2005 : 259).

43 C'est ce que m'expliqua le gouverneur du district de Jéricho : " Dans la culture palestinienne, tout le monde veut partir tôt, tout le monde veut être au premier rang et dans le premier bus. Et ça fait de la pression pour les gens qui travaillent à l'istiraha [...]. On essaie de convaincre les gens de changer leurs horaires de voyage. Mais bon, le cerveau palestinien n'est pas toujours évident à contrôler [il sourit] " (Jéricho, juin 2011). 


\title{
Des facilitateurs de passage : les chauffeurs de taxi
}

\begin{abstract}
Juillet 2011. J'accompagne Sara et sa fille Leila, qui partent à Amman pour les vacances, jusqu'à l'istiraha de Jéricho. Quand le taxi passe me chercher, il est 4 heures du matin. Leila et sa mère sont installées à l'arrière. Le chauffeur parle au téléphone : "Dans cinq minutes je suis chez toi ". Un homme attend au pied d'un immeuble, une valise posée sur le sol. Le chauffeur descend, s'empare de la valise et la dépose dans le coffre. Avant de démarrer, il raye un nom sur sa liste. "Passeport ? Permis ? Carte ? Tout va bien (kullshî tamâm) ? " Le passager acquiesce en palpant une nouvelle fois son portefeuille dans sa poche. Une centaine de mètres plus loin, le chauffeur téléphone à nouveau : "Vous êtes prêts ? J'arrive dans cinq minutes". Trente minutes plus tard, les passagers sont au complet. Le minibus approche de la sortie de la ville : " S'il vous plaît, tout le monde attache sa ceinture ", annonce le chauffeur, désireux d'éviter une amende de la police israélienne sur la route qui sépare Naplouse de Jéricho. Quand nous arrivons à destination, le soleil se lève. Le taxi prend sa place dans la file de voitures qui attendent devant la salle de I'istiraha. Après avoir acheté un café à un marchand ambulant, nous remontons en voiture, la porte s'ouvre et nous entrons dans la zone. Le chauffeur nous explique qu'on va nous donner un ticket : Sara et Leila ont les numéros 51 et 52 . II leur précise qu'elles seront dans le deuxième bus ${ }^{44}$.
\end{abstract}

Ces scènes de départ se reproduisent tous les matins pendant l'été, période $d^{\prime}$ affluence au pont Allenby. Les chauffeurs de taxi ${ }^{45}$ sont les premières personnes auxquelles les passagers ont à faire : ils rassemblent les voyageurs, s'assurent que ces derniers ont tous les documents nécessaires et s'occupent des bagages. Puis il faut choisir la route pour se rendre à Jéricho. Abu Fadi, chauffeur depuis une dizaine d'années, me dit :

"Nous les chauffeurs on essaie toujours de prendre la route la plus courte. Certains passagers vont te dire: "Ne passe pas par une route où il y a des checkpoints". Sur la route des mu'arrajât ${ }^{46}$, il n'y a pas de checkpoints. Si je prends la route de Maale Efrayim $^{47}$, je vais devoir m'arrêter et être fouillé. Donc toi, si tu as un avion à prendre, tu vas me dire: "Je ne veux pas passer par des checkpoints, je veux une route rapide" [...] et moi, je suis bien obligé de prendre la route que veulent les passagers" (Naplouse, janvier 2011).

Les chauffeurs font le point sur l'état du trafic, sur une question d'un passager ("Kif al-jisr? ", " Comment est le pont ? ") ou de leur propre initiative.

\footnotetext{
44 Extrait de mes notes de terrain, 2011.

45 Dans chaque ville de Cisjordanie, plusieurs compagnies proposent des services de transport collectif pour l'istiraha. Selon les saisons et l'affluence, le transport se fait dans des voitures à cinq places ou des minibus à huit places. Le trajet depuis Naplouse jusqu'à Jéricho est fixé à 50 shekels israéliens (10 euros), 30 depuis Ramallah.

46 Littéralement route des " tournants ", c'est une route à flanc de coteau avec de nombreux lacets.
}

47 II s'agit d'une route qui passe devant une colonie israélienne, et récemment ouverte aux voitures palestiniennes. Elle est réputée plus sûre que la route des mu'arrajât. 
II s'agit de préparer les voyageurs à franchir un pont bondé (meliân ou azmeh ${ }^{48}$ ) ou au contraire, de les rassurer s'il est " vide " (fâdi). Ils signalent les changements d'horaires ou les fermetures anticipées, désamorcent les rumeurs ("il paraît que le pont ferme à 16 heures aujourd'hui ? "). Certains tiennent à disposition des passagers les fiches qu'ils devront remplir à leur entrée en Jordanie. Ils connaissent les bureaux où ceux qui en ont besoin pourront se faire établir un permis de sortie. En été, quand la file des voitures devant l'istiraha s'étend parfois sur un kilomètre, les chauffeurs rassurent les passagers : "Cela va aller vite, vous aurez une place dans le bus ". À l'arrivée dans l'enceinte de la zone, ils expliquent les différentes étapes : où acheter les billets de bus, où payer la taxe de sortie, où laisser les bagages. Ils prodiguent aussi des conseils : prendre une bouteille d'eau, ne pas donner de pourboire aux porteurs, etc.

\section{Négocier les contrôles}

Lors des passages au guichet, notamment dans la salle d'arrivée israélienne, il s'agit de garder son calme et de ne pas attirer l'attention, afin de passer le plus rapidement possible. Les passagers cherchent généralement à réduire au minimum les interactions avec les autorités, de façon à faire du contrôle des papiers une simple "formalité " et éviter qu'il ne conduise à un interrogatoire surtout, que ce dernier ne se solde par un refoulement. Les voyageurs tentent d'ajuster leurs comportements, selon que l'on a à faire à un homme ou une femme, ou à tel ou tel employé déjà rencontré lors d'un précédent passage et qui a été " bien " (mnîh), " humain " (insânî), " compréhensif " (fahmân). S'il arrive qu'un employé tente une plaisanterie, chacun sait bien que la marge de négociation est très faible étant donnée l'asymétrie de la situation. J'assistai lors d'un de mes passages à une conversation entre un vieux monsieur palestinien (muni d'un passeport américain) et un agent israélien d'une cinquantaine d'années :

" [L'agent israélien :] - Quelle est la raison de ta visite en Israël? - Je ne vais pas en Israël, je vais voir ma fille à Ramallah.

- Tu n'as que ce passeport? Je vois sur l'ordinateur que tu as une carte d'identité (hawiyya) palestinienne. - Non, je l'ai perdue il y a longtemps.

- L'ordinateur me dit que tu as un numéro de hawiyya. [En riant] Et en effet, ça fait très très longtemps que tu l'as perdue! Tu voudrais la récupérer?

- Oui, bien sûr, est-ce que c'est possible?

- Tsss tss... [II fait un geste du menton en signe de dénégation] Pourquoi, tu n'aimes pas les États-Unis? Bon allez, va t'asseoir, tu vas attendre un peu.

- Longtemps? Combien de temps?

- Un peu je te dis. Allez, va t'asseoir.

- Mais je vais attendre combien de temps?

- Tu attends qu'on t'appelle $»^{49}$.

48 Meliân veut dire " plein " par opposition à " vide " (fâdî). Le mot azmeh signifie " crise ". Pendant les heures de pointe, on dira volontiers " fi azmeh 'al jisr ", littéralement " C'est la crise au pont".

49 Extrait de mes notes de terrain, janvier 2013. 
Avec les autorités palestiniennes et jordaniennes, on retrouve des configurations comparables. Cependant, des connaissances personnelles voire des connivences peuvent éventuellement être activées, ce qui permet d'accélérer le déroulement des contrôles - et le passage de manière générale.

\section{Maîtriser l'enchaînement des procédures, faire jouer " ses relations ", payer des bakchichs}

Karim, qui se rend régulièrement en Jordanie pour rendre visite à sa famille, raconte son passage vers Amman, un jour de pèlerinage :

" Je suis arrivé à lístiraha à midi moins le quart, ils m'ont donné le numéro 3728. Je suis entré, il y avait beaucoup de monde. [...] J'ai appelé Maya ${ }^{50}$, qui a appelé sa copine qui travaille à l'istiraha, elle s'appelle Oum Husam. [...] Elle m'a dirigé vers quelqu'un

d'autre. Je suis allé le voir, mais il n'avait plus de numéros. Je pense qu'ils ont des numéros en stock, pour donner à des gens comme moi... Mais là, ça n'a pas marché, il

n'y en avait plus. [...] Donc, j'ai dû attendre comme ça trois heures et demie. Ensuite, on a changé de bus à al-'alami, comme d'habitude. Donc moi je monte devant... Parce que je fais toujours ça, je cours, comme ça je monte le premier dans le deuxième bus. Je n'ai que mon petit sac, je le pose sur la table, hop, comme ça je suis le premier. [...] Ensuite, on prend encore un bus, pour aller chercher les bagages. Donc moi, je me prépare parce que j'ai beaucoup d'expérience, je me mets derrière la porte comme ça je peux être le premier à sauter et acheter mes tickets " (entretien en français, Amman,

2012).

Karim est un voyageur averti qui déploie différentes " techniques " pour minimiser les temps d'attente. La première consiste, quand il y a foule (azmeh), à faire jouer ses relations personnelles pour obtenir un traitement de faveur. Si on connaît quelqu'un (ou quelqu'un qui connaît quelqu'un) qui travaille à la police palestinienne ou à l'administration des passages, on peut récupérer un numéro qui facilite la montée dans le bus. La deuxième s'appuie sur sa connaissance de l'enchaînement des procédures. Elle consiste à s'installer sur les sièges avant (voire rester debout) afin d'être le premier à descendre du bus et à acheter son billet pour le suivant. Plus que les précieuses minutes ainsi gagnées, il s'agit surtout de rétablir une marge d'action personnelle donnant le sentiment d'une maîtrise, dans les interstices d'un dispositif spatio-temporel imposé par les autorités de contrôle. Karim (comme bien d'autres voyageurs) fait ici preuve d'une " habileté technique en contexte, une forme de connaissance pratique manifestant une intervention active de l'acteur "(Breviglieri, 2004 : 19) : elle engage un savoir-faire informel, qui s'appuie sur des connaissances établies au fil des passages (ou délivrées par d'autres, sous forme de conseils). L'activation des relations personnelles n'est cependant pas toujours couronnée de succès, et le déroulement du voyage est souvent perturbé.

Un jour de grande affluence (c'était la fin des fêtes de l'aïd ${ }^{51}$ ), je passais le pont avec Karim depuis Amman vers la Cisjordanie :

50 II s'agit de la femme de l'ancien gouverneur adjoint de Naplouse, une collègue de Karim.

51 L'aïd al-fitr (fête de la rupture du jeûne) commémore chaque année la fin du mois de Ramadan. 
Le bus stationne devant le terminal israélien. [...] Le chauffeur dit aux passagers de descendre par la porte arrière. Cela irrite Karim, qui s'était assis exprès près de la porte avant. Pendant que nous descendons, les bagages ont été débarqués par des porteurs. Karim me dit qu'il va récupérer les valises, puis payer un porteur pour qu'il les fasse passer devant tout le monde ${ }^{52}$.

Comme Karim, Diana (originaire de Naplouse) distribue à l'occasion quelques bakchichs ${ }^{53}$ aux porteurs et aux chauffeurs de bus. Elle me raconta son voyage vers Amman, un jour de pèlerinage :

"Quand nous sommes arrivés du côté jordanien, il y avait cinq bus devant nous! Je me suis dit: "On ne va pas attendre", je suis allée voir le chauffeur et je lui ai donné 10 dinars en lui disant que je devais prendre l'avion dans la journée... et il nous a emmenées au premier bus".

En effet, en cas de queue sur le pont, les chauffeurs jordaniens acceptent souvent (dans un sens et dans l'autre) de faire passer un passager plus ou moins discrètement jusqu'au premier bus, moyennant pourboire ; ce que j'ai pu observer lors de ma traversée avec Karim, d'Amman vers la Cisjordanie.

Nous arrivons sur le pont, il y a sept bus devant nous. Certains
passagers se lèvent, parlementent avec le chauffeur. [...] S'ils n'ont
pas de bagage dans le coffre, il les fait sortir par le bas-côté et
les emmène jusqu'au premier bus. L'important est que le policier
jordanien, installé dans une cahute à côté du dernier checkpoint ne
puisse pas les voir facilement. Plusieurs passagers changent ainsi
de bus. ${ }^{54}$

Si gagner du temps génère toute une économie informelle, certains passagers n'hésitent pas à " gruger ", doubler la queue ou encore simuler un mauvais état de santé, estimant qu'ils ne font que rétablir leur bon droit. D'autres, à l'inverse, insistent sur la nécessité d'une discipline irréprochable et d'une observation parfaite des "règles " du passage. Enfin, tous les Palestiniens ne passent pas le pont de la même façon. Un voyageur pressé, et surtout qui en a les moyens peut, du côté jordanien, acheter le temps de son passage auprès de la compagnie al-Sahm (la flèche) pour un service appelé "VIP ». II deviendra ainsi, jusqu'au côté israélien, un "VIP " temporaire ${ }^{55}$. Contre 50 dollars, il attendra en buvant du café dans la " salle VIP " qu'un employé jordanien s'occupe de ses formalités de départ. II sera ensuite acheminé dans un van, sans attendre aux checkpoints et sans subir de fouille, jusqu'au côté israélien.

\section{Observation sur le pont, vers la Cisjordanie, 2010.}

53 Le montant de ces bakchichs est généralement modique, et à la différence du service "VIP " mentionné plus bas, ne dessine pas de différenciation " de classe " entre les Palestiniens qui passent le pont.

54 Observation sur le pont, vers la Cisjordanie, 2010.

55 L'appellation " VIP " désigne en principe le personnel de l'Autorité palestinienne, mais aussi des commerçants titulaires de la BMC (Business Man Card). Les accords d'Oslo prévoient des " arrangements spéciaux " pour faciliter le passage de ces VIP. Pour se rendre en Jordanie, ils ne passent pas par l'istiraha; ils sont attendus au pont par des agents israéliens qui les font passer devant les autres passagers, puis ils prennent un taxi jusqu'au terminal jordanien (Bontemps, 2012). 
Dans les bus, aux queues devant les guichets, dans les salles, la plupart des Palestiniens " ordinaires " tentent, quant à eux, d'occuper les temps de l'attente et de rendre les lieux " habitables ". Ils lisent le journal, téléphonent à leurs proches, jouent avec leur téléphone portable, parfois dorment et bien souvent conversent avec leurs voisins. Échange de paroles, d'informations, de plaisanteries, toutes formes de rapports peuvent se nouer au gré des circonstances du passage. Si ceux-ci sont marqués par l'anonymat propre aux espaces de transit (gares, aéroports), ils n'en constituent pas moins une façon de se familiariser avec les espaces-temps de la traversée. Les longues attentes dans des espaces bondés sont associées à la tension, à la bousculade, à la pollution sonore ou aux cris des enfants. L'épreuve dans laquelle les passagers se trouvent " embarqués " favorise pourtant la parole et la formation de liens faibles. L'autre n'est plus un obstacle, mais devient " un compagnon de galère ", voire un allié. Les sociabilités ponctuelles qui se créent peuvent alors donner le sentiment de résister, avec les moyens du bord, au dispositif frontalier.

\section{Des micro-sociabilités en voyage}

Lors du passage du pont, le contexte d'une épreuve commune permet la cristallisation d'un collectif éphémère. Celui-ci se forme et dure le temps du voyage (safar) - sorte de parenthèse dans la marche des vies de chacun, à travers ses différentes étapes : dans le taxi collectif, dans les queues, dans les bus, enfin dans les salles d'attente ${ }^{56}$.

Tout commence dans le taxi qui transporte les passagers vers Jéricho : la conversation s'engage souvent très vite à propos de l'organisation des bagages, de leur contenu, des courses à faire, des souvenirs à acheter, etc. La plupart des passagers voyagent très chargés et transportent des vêtements, de la nourriture, des cadeaux (souvent de l'huile d'olive du pays). On discute aussi du meilleur trajet à emprunter jusqu'à Jéricho. Puis tout au long de la traversée, au hasard de la personne à côté de laquelle on se retrouve assis dans le bus ou dans les salles, se mettent en place des "sociabilités minimalistes " (Puig, 2013) qui s'appuient sur différents ressorts, et ont diverses fonctions. Échange d'informations et de renseignements, tout d'abord : combien coûte maintenant la taxe de sortie ? Combien faut-il payer aux Jordaniens à I'arrivée ? Où acheter son ticket de bus ? Les voyageurs bien rodés font profiter les autres de leur expérience. On rend des services, on s'entraide en portant les bagages, en prêtant de l'argent, etc. Aux abords des guichets, certains en aident d'autres à parlementer en anglais avec un employé israélien qui ne parle pas l'arabe.

Un autre ressort de ce collectif éphémère consiste en commentaires échangés sur la situation. On se raconte les passages antérieurs, les expériences vécues: " Je me souviens, la dernière fois ils m'ont fait attendre trois heures "; " je me souviens, c'était la même personne au guichet, elle a été très désagréable avec moi " (ou au contraire, "plutôt sympa "). On raconte des anecdotes et on

\footnotetext{
56 De nombreux auteurs dont Pétonnet se sont intéressés à ces situations où le dialogue devient licite dans les espaces publics, à la faveur d'une " entrée en matière d'emblée reconnue comme telle [...] une circonstance, une particularité, une situation, voire un lieu quelque peu exceptionnels ou insolites " (Pétonnet, 1987 : 254-255). Elle évoque, dans le contexte parisien, une grève des transports, un arrêt de la rame du métro, la présence d'une ambulance ou d'une voiture de pompiers dans la rue.
} 
plaisante, certes pour passer le temps, mais aussi pour mettre à distance les événements, l'inconfort ou l'humiliation subis.

\begin{abstract}
$\overline{A u ~ " ~ p o n t ~ i s r a e ́ l i e n ~ ", ~ s a l l e ~ d ' a r r i v e ́ e . ~ J ' a t t e n d s, ~ a s s i s e ~ e t ~ j ' o b s e r v e ~}$ les passagers qui font la queue devant les guichets. Une famille s'avance, un couple avec plusieurs enfants en bas âge, dont deux bébés. L'employée israélienne prend les papiers que lui tend le père de famille, elle s'évertue à vérifier si chaque bébé correspond bien à la photo sur le passeport. Elle s'énerve un peu : "Où est Mohammad? Et laquelle est Nisrine? " Les femmes se mettent à rire, l'employée est obligée de quitter son guichet et n'arrive que difficilement à identifier les enfants. Un peu plus tard, toute la famille va s'asseoir, plaisantant avec leurs voisins sur ce qui vient de se passer ${ }^{57}$.
\end{abstract}

Ces sociabilités sont ponctuelles et fragiles, tandis que le dispositif frontalier tend à séparer les catégories de passagers (Bontemps, 2012). Si la foule est trop importante et si des bousculades se produisent, les disputes éclatent. Lors des entretiens ou dans nos conversations, certains Palestiniens disent éprouver de la gêne ou de la honte devant le manque de discipline de leurs compatriotes qui perdent leur sang-froid, recourent au bakchich ou au mensong $\mathrm{e}^{58}$. Les petits liens et les petites alliances ponctuelles peuvent vite se défaire dès lors que l'autre devient une menace, notamment de faire " perdre du temps".

\title{
Arrivé à destination : refaire le voyage
}

Une fois arrivés en Jordanie (le plus souvent à Amman), certains passagers vont à l'hôtel dans l'attente de leur vol du lendemain ; mais la plupart se rendent dans leur famille. Les taxis collectifs jordaniens les y déposent, parfois au prix de très longs détours dans les périphéries tentaculaires de la capitale. Le voyage se prolonge souvent après l'arrivée : les voyageurs racontent leur passage aux membres de leur famille (c'était " calme ", " bondé ", etc.), décrivent l'attitude de tel ou tel soldat ou signalent une rencontre inattendue, exagèrent parfois les temps d'attente (" J'ai attendu huit heures, dix heures ") ou enjolivent une réponse ("Tu ne sais pas ce que je lui ai dit ? "). Ces récits tendent aussi à réaffirmer une légitimité et un droit au passage, malgré l'humiliation ressentie. Dans leurs exagérations mêmes, ils peuvent être vus comme des " petits actes réparateurs " (Puig, 2012 : 153), une façon de réajuster symboliquement une situation pour revenir, après la dureté du passage, vers la vie "normale ".

\section{Conclusion}

Dans le contexte de rupture des accords d'Oslo qui fait suite à la deuxième Intifada, la succession des procédures au pont Allenby reflète avant tout un rapport de force entre les parties en présence, ainsi que la manière dont l'occupation israélienne entend (en coopération avec l'État jordanien et l'Autorité palestinienne) contrôler les mobilités des Palestiniens. L'épisode de la mort du juge Zuaiter a montré que si ces procédures apparaissent aujourd'hui bien réglées à travers les étapes d'un circuit balisé, le pont reste un espace sous tension, où

57 Extrait de mes notes de terrain, mars 2011.

58 Romani (2005 : 266) fait la même constatation à propos du passage des checkpoints. 
les contrôles peuvent " déraper ". Plus précisément, cet événement rend visible I'exercice de la domination ordinaire subie par les Palestiniens au pont Allenby. II faut rappeler que nombre d'entre eux ne peuvent pas franchir le pont, et que ceux qui pourraient tenter la traversée (en demandant un visa à l'ambassade d'Israël à Amman par exemple) hésitent souvent à le faire : ils refusent d'accorder une légitimité à cette frontière, ou encore redoutent l'épreuve que représente un passage avec lequel ils ne sont pas familiers. Le rapport avec un soldat israélien leur apparaît insupportable, alors que pour des passagers " habitués" ces contrôles font aujourd'hui partie d'une " routine " (rûtîn).

Dans ces conditions, les tactiques mises en œuvre par les passagers au pont apparaissent sous un jour paradoxal. Formées avec l'usage, elles permettent aux " traversants " de s'approprier en partie l'espace et les temporalités du passage. Ils reconstruisent une rationalité dans l'épreuve de la domination, à travers des " routines " de franchissement et des formes d'échanges sociaux spécifiques: celles-ci permettent de composer avec la violence de la traversée, voire d'habiter le lieu. Mais dans le même temps, ces "routines " garantissent le " bon déroulement " des procédures de contrôle : intériorisées avec la longue expérience de la relation occupant-occupé, elles peuvent être comprises comme des pratiques d'autocensure ou d'autodiscipline ${ }^{59}$.

\section{Références bibliographiques}

Ababsa Myriam et Daher Rami (Éds.) (2011) Villes, pratiques urbaines et construction nationale en Jordanie, Beyrouth, Presses de I'Ifpo, 328 p.

Agier Michel (2013) La condition cosmopolite. L'anthropologie à l'épreuve du piège identitaire, Paris, La Découverte, $212 \mathrm{p}$.

Balibar Étienne (1997) La crainte des masses. Politique et philosophie avant et après Marx, Paris, Galilée, $456 \mathrm{p}$.

Bonnefoy Laurent, Bontemps Véronique et Guignard Xavier (2012) La Palestine en réseaux : une approche en cours de redéfinition, Les Carnets de I'lfpo, [en ligne] consulté le 14/01/2014. URL : http://ifpo.hypotheses.org/4282

Bontemps Véronique (2012) Le temps de traverser le pont, Temporalités, 15, [en ligne] consulté le 06/06/2012. URL : http://temporalites.revues.org/1995

Breviglieri Marc (2006) Le fond ténébreux de la routine. À propos des morales du geste technique au travail, in Sandra Laugier et Claude Gautier Éds., L'ordinaire et le politique, Paris, PUF/CURAPP, pp. 189-217.

Breviglieri Marc (2004) Habiter l'espace de travail. Perspectives sur la routine, Histoire et sociétés, 9, pp. 18-29.

De Certeau Michel (1990) L'invention du quotidien. Vol. 1/2. Arts de faire, Paris, Gallimard, $349 \mathrm{p}$.

59 Cela ressort particulièrement bien dans les entretiens, lorsque les passagers évoquent la volonté des autorités israéliennes de les dresser les uns contre les autres, ou celle des Jordaniens de favoriser des pratiques de "resquille ", qui fragilisent I'expérience du collectif palestinien. 
Havkin Shira (2011) La privatisation des checkpoints : quand l'occupation militaire rencontre le néolibéralisme, in Stéphanie Latte Abdallah et Cédric Parizot Éds., $\dot{A}$ I'ombre du mur. Israéliens et Palestiniens entre séparation et occupation, Arles, Actes Sud, pp. 51-72.

Kuttab Daoud (2014) Israeli Occupier-Occupied Paradigm Must End, [en ligne], consulté le 14/03/2014. URL : http://linkis.com/huff.to/NfqoA

Lemieux Cyril (2007) À quoi sert I'analyse des controverses ?, Mil neuf cent, Revue d'histoire intellectuelle, 25, pp. 191-212.

Lussault Michel (2013) L'avènement du Monde. Essai sur I'habitation humaine, Paris, Seuil, 296 p.

Lussault Michel (2012)Trans-spatialités urbaines, Hermès, La Revue, 63, pp. 67-74.

Parizot Cédric (2009)Temporalités et perceptions de la séparation entre Israéliens et Palestiniens, Bulletin du Centre de recherche français à Jérusalem, 20, [en ligne] consulté le 13/06/2011. URL : http://bcrfj.revues.org/6291

Perdigon Sylvain (2010) L'ethnographie à I'heure des martyrs, Annales. Histoires, Sciences Sociales, pp. 971-996.

Peteet Julie (2008) Stealing Time, Middle East Report, 248, pp. 14-17.

Pétonnet Colette (1987) L'anonymat ou la pellicule protectrice, in Collectif, La ville inquiète, Paris, Gallimard, pp. 247-261.

Puig Nicolas (2013) Lost in Transition: Ordeals of Passage of Palestinian Refugees in Lebanon, Mediterranean Politics, 18 (3), pp. 394-410.

Puig Nicolas (2012) Villes intimes. Expériences urbaines des réfugiés palestiniens au Liban, in Mohamed Kamel Doraï et Nicolas Puig Éds, L'urbanité des marges. Migrants et réfugiés dans les villes du Proche-Orient, Paris/Beyrouth, Éditions Ifpo/Téraèdre, pp. 235-256.

Romani Vincent (2005) Quelques réflexions à partir des processus coercitifs dans les Territoires occupés, Études rurales, 173-174, pp. 251-272.

Weizman Eyal (2007) Hollow Land: Israel's Architecture of Occupation, London, Verso, $318 \mathrm{p}$. 


\section{Véronique Bontemps}

\section{Entre Cisjordanie et Jordanie, l'épreuve du passage frontalier au pont Allenby}

Cet article décrit la manière dont les Palestiniens vivent et rendent compte de leur expérience frontalière, lorsqu'ils passent le pont Allenby entre la Cisjordanie et la Jordanie. L'auteure montre que ce passage représente pour eux une expérience pénible et éprouvante, mais pourtant relativement ordinaire : ce qu'elle propose d'appeler une "épreuve routinière ". Après un rappel historique montrant comment s'est formé l'espace frontalier au pont, l'article expose les différents niveaux de cette "épreuve " telle qu'elle est perçue par les Palestiniens (violence de l'attente, de l'incertitude et de l'arbitraire), ainsi que les " techniques " que ces derniers développent pour diminuer l'inconfort de la traversée et la rendre " acceptable ".

\section{Between the West Bank and Jordan, the Ordeal of Border-crossing at the Allenby Bridge}

In this paper the author describe how Palestinians cross the Allenby Bridge between the West Bank and Jordan, and how they talk about it. She shows that this crossing represents for them a painful and trying, but at the same time relatively common experience: which the author proposes to call a "routine ordeal". After a historical outlook in order to show how the border zone was shaped at the Bridge, the article focus on the crossing itself as experienced today by Palestinians, and the feelings they express when talking about it (especially the violence of waiting, of uncertainty and of arbitrariness). Finally, the author addresses the various "techniques" they develop in order to minimize the suffering of the crossing and make it "acceptable".

\section{Entre Cisjordania y Jordania, las penalidades del paso fronterizo del puente Allenby}

Este artículo describe cómo los palestinos viven y dan cuenta de su experiencia fronteriza, cuando pasan el puente Allenby entre Cisjordania y Jordania. El autor muestra que este paso representa para ellos una experiencia dolorosa y difícil, sin embargo relativamente corriente, por lo que propone denominarla "penalidad rutinaria». Después de un recordatorio histórico de cómo se formó la zona fronteriza con el puente, el artículo aborda los diferentes niveles de esta "prueba» según la percepción de los palestinos (la violencia de la espera, la inseguridad y la arbitrariedad), así como las "técnicas" que estos han ido desarrollando a fin de reducir el malestar de la travesía, convirtiéndola en «aceptable». 